\title{
Comparison Between Configurational Bias Monte Carlo and Molecular Dynamics Sampling for Sampling Conformational Stability with High Energy Barriers
}

Henrique Musseli Cezar ${ }^{1 *}$ (PG), Sylvio Canuto ${ }^{1}$ (PQ), Kaline Coutinho ${ }^{1}$ (PQ) henrique.cezar@usp.br

${ }^{1}$ Instituto de Física, Universidade de São Paulo, Rua do Matão No 1371 CEP 05508-090 Cidade Universitária, São Paulo

Palavras-chave: Monte Carlo, Conformational Sampling, syn/anti stability, mesityl oxide.
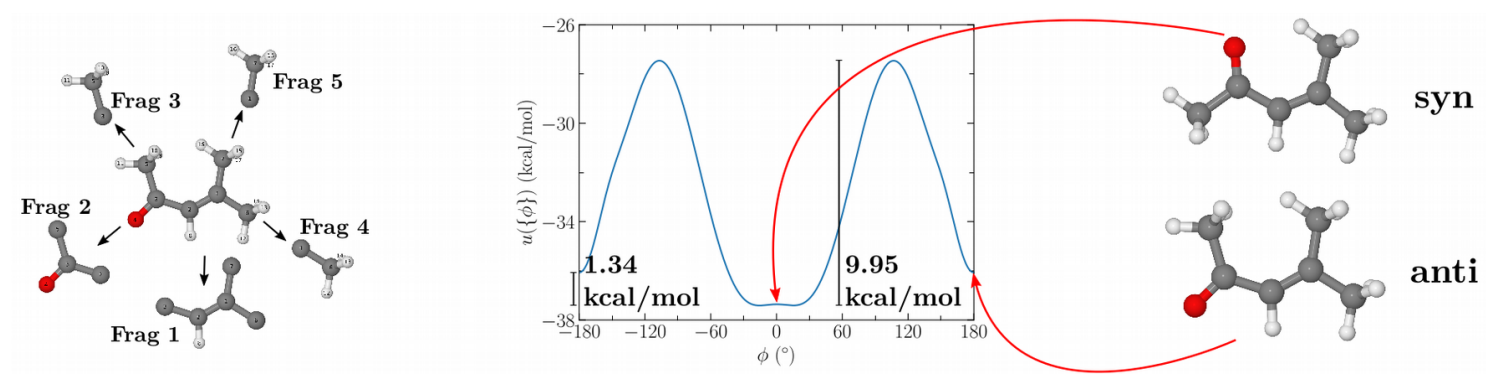

Fig 1: Fragmentation scheme of MOx used for the CBMC algorithm, together with the torsional potential for the rotation defining the syn and anti conformers. Figure from Ref. [2].

The sampling of intra molecular degrees of freedom is important because several properties are dependent on the molecular geometry. For molecules that can have more than one conformation, it is known that the conformational stability can be solvent dependent, and that the stable conformations are separated by energy barriers. There are several examples in the literature of molecules that have population ratios changed dependent on the environment. Some of these molecules have large rotational barrirers (10 kcal/mol or larger) between the conformations. In the literature, studies comparing Monte Carlo (MC) and Molecular Dynamics (MD) methods to sample conformation around smaller barriers (about 3-4 kcal/mol) have been performed, concluding that both methods achieve similar sampling, but larger barriers have not been addressed. One example of molecule with two stable conformations separated by a barrier larger than the $3-4 \mathrm{kcal} / \mathrm{mol}$ is the mesityl oxide $(\mathrm{MOx})$ molecule $\left[\left(\mathrm{CH}_{3}\right)_{2} \mathrm{C}=\mathrm{CHC}(=\mathrm{O}) \mathrm{CH}_{3}\right.$, 4methyl-3-penten-2-one]. The $\mathrm{MOx}$ is a model for larger species of ketones, characterized by a carbonyl group that connected to two other organic groups, with a single bond in the middle. The rotation around this single bond generate the syn and anti conformations, shown in Fig 1. Free energy calculations [1] have shown that in gas phase, the syn conformation is more stable, while in water the populations change and the MOx anti conformation becomes dominant. In this work,[2] we parametrize the MOx torsionals to reflect the gas phase energy differences, and then perform Configurational Bias Monte Carlo (CBMC) and MD simulations to investigate how the populations are sampled in each methodology. We show that traditional MD, i.e., MD without enhanced sampling techniques, has problems overcoming the $\sim 10 \mathrm{kcal} / \mathrm{mol}$ energy barrier separating each conformer, while CBMC samples the expected populations. For gas phase, a population of 91:9 \% of syn:anti conformations is expected,[1] a value very close to the one obtained with the CBMC method that we have implemented in DICE, of 95:5 \%. In water, the syn:anti population obtained with CBMC changes to $25: 75 \%$, which is in agreement with the trend obtained by the free energy calculations.[1] The conformer populations obtained with MD are strongly 
dependent on the initial conditions, both in gas phase and water, achieving only few changes between one conformer and the other. We conclude that the MD sampling for molecules with high energy barriers (of $\sim 10 \mathrm{kcal} / \mathrm{mol}$ or more) must be performed with caution and point the CBMC method as an alternative.

[1] Damasceno et al (2012). Theor. Chem. Acc., 131(5), 1214. DOI: 10.1007/s00214012-1214-y

[2] Cezar et al (2018). Int. J. Quantum Chem. In Press. DOI: 10.1002/qua.25688 